\title{
Embodiment: A Brief Overview
}

\author{
Mansoor Fahim \\ Allameh Tabataba'i University, Tehran, Iran \\ E-mail: dr.manfahim@yahoo.com \\ Samaneh Eslamdoost (Corresponding author) \\ Allameh Tabataba'i University, Tehran, Iran \\ E-mail: samaneh.eslamdoost@gmail.com
}

Received: 22-06-2014

doi:10.7575/aiac.ijalel.v.4n.1p.73
Accepted: $30-08-2014$

Published: 01-01-2015

URL: http://dx.doi.org/10.7575/aiac.ijalel.v.4n.1p.73

\begin{abstract}
One of the bewildering conceptions within the realm of cognitive science has been the perception of meaning and understanding, which has inadequately been approached by previous theories of cognition. Recently, there have been radical shifts in developments in cognitive science toward the theory of Embodiment. On the word of this newfangled standpoint, it is widely accepted that body plays a central role in meaning making. In this novel approach, making linguistic meanings which is closely related to our actions is precisely the point. Several investigations have endeavored to provide the estimates of embodiment by examining the effect of body movements on cognition. A considerable amount of literature has proved that the cognitive capacities are constructed and dependent on the bodily actions through the interaction with the external world.
\end{abstract}

Keywords: Embodiment, Embodied Cognition, Meaning Perception

\section{Introduction}

One of the most significant discussions in Philosophy has been dealing with the issue of the meaning and where it comes from. Accordingly, more recent attention has focused on the provision of the concept of meaning making to be investigated in cognitive science. Human beings have capacities for percept based and concept based thinking. According to Embodiment Theory, these capacities are closely linked to the sensorimotor capability which can be assumed to be in sharp contrast with language as an autonomous language faculty approach. In this view, it is largely believed that conceptual and linguistic representation is basically the result of reprocessing sensorimotor representational capacities.

Embodiment perspective is quite in contrast with the traditional notion of cognition which highlights the role of cognition as to be internal, symbolic, computational, and disembodied (Gibbs, 2003). Within traditional view of cognition, comprehension of linguistic input constitute a process of mapping words onto the representations of their meaning which ultimately leads to capturing the content meaning, that is understanding is the same as producing the semantic meaning. The aim of the paper is to provide a conceptual theoretical framework based on different closely related aspects of the concept of Embodiment. The first intention of the present study is to try to put forward a thorough definition of Embodiment. Subsequently, drawing upon the stand of research into the new approach toward cognition, this study attempts to clarify several aspects of Embodiment encompassing Embodiment and the semantic meaning, perception of the reality, linguistic comprehension, conceptual processing, and the role of the environment. And finally, a part of the aim of this study is to shine light on these debates by presenting the evidence and claims of the Embodiment provided in the body of the literature.

\section{What Is Embodiment?}

One of the pioneering elaborations on the concept of Embodiment comes from Merleau-Ponty and Heidegger (cited in Anderson, 2003, p. 104).

Heidegger, for instance, shows - especially in his celebrated analysis of being-in-the world - that the condition of our forming disengaged representations of reality is that we be already engaged in coping with our world, dealing with the things in it, at grips with them. . . It becomes evident that even in our theoretical stance we are agents. Even to find out about the world and formulate disinterested pictures, we have to come to grips with it, experiment, set ourselves to observe, control conditions. But in all this, which forms the indispensable basis of theory, we are engaged as agents coping with things. It is clear that we couldn't form disinterested representations any other way. What you get underlying our representations of the world - the kinds of things we formulate, for instance, in declarative sentences - is not further representations but rather a certain grasp of the world that we have as agents in it [114, pp. 432-433].

Accordingly, in line with the preceding definitions of Embodiment, Wilson and Golonka (2013) proposed that "Embodiment is the surprisingly radical hypothesis that the brain is not the sole cognitive resource we have available to 
us to solve problems. Our bodies and their perceptually guided motions through the world do much of the work required to achieve our goals, replacing the need for complex internal mental representations. This simple fact utterly changes our idea of what "cognition" involves, and thus embodiment is not simply another factor acting on an otherwise disembodied cognitive processes" (p.1).

Still, many scholars find the above definition as biased and put forward their concern as how cognition can be totally biased by motions and states of body? (Shapiro, 2011).

\section{Embodiment and Semantic Meaning}

As cited in Sinha and Lopez (2000), "the Embodiment thesis is central to cognitive semantics, both as a general philosophical and psychological perspective, and as a source of specific hypotheses about linguistic meaning, metaphor, imagination and cognitive mappings (Johnson, 1987; Lakoff, 1987; Lakoff \& Johnson 1999)" (p.17). Embodiment, at first stage, challenges the notions of Cartesian Dualists and deeply focuses on interrelatedness of the bodily actions from the very beginning with cognition, which is quite in line with existing concerns in cognitive neuroscience (Damasio, 2000) as well as epigenetic connectionist computational modeling (Elman et al., 1996; Plunkett and Sinha, 1992).

Over the past decades, there have been rapid advances on the representation of the cognition as a coupled process between the internal and the external in cognitive science. As it has been an increasing concern, Kerkhofs and Haselager (2006, p. 1) raise the issue of semantic representation which has been put forward by Harnad (1990) who has formulated the basic question as "How can the semantic interpretation of a formal symbol system be made intrinsic to the system, rather than just parasitic on the meanings in our heads? (...). The problem is analogous to trying to learn Chinese from a Chinese/Chinese dictionary alone". As a matter of fact, "for Harnad meaning has to be grounded in something different than just other (equally meaningless) symbols".

\subsection{Embodiment and Linguistic Meaning}

One question that needs to be answered is that how Embodiment is connected to the meaning? Recently, in vitro studies (Barsalou, 1999; Glenberg, 1997; Zwaan, 2004) have shown that Embodiment is based on the idea that embodied meaning, which is in contrast to the typical order of the linguistic stimulus, has a major role in perception. Latest evidence suggests that the comprehender perceives the external models and internalizes the patterns of meaning which leads to the internal perception. In this regard, seeing a word, an individual activates all the perceived elements that has been exposed to from the external world, such as the shape, weight, smell, color, etc., which paves the way for the perception of the meaning of that particular word (Pecher \& Zwaan, 2005; Semin \& Smith, 2008). It has conclusively been shown that if there is a direct correspondence between the word and the meaning the comprehension of the meaning is much easier. In contrast, data demonstrated that when the iconicity between the word and the meaning, which can be seen in words for animals, motion verbs, and object words, is not established, the process of meaning perception turn out to be perplexing (Estes, Verges, \& Barsalou, 2008; Meteyard, Baharami, \& Vigliocco, 2007; Richardson \& Spivey, 2000; Šetic \& Domijan, 2007), (all cite in Louwerse \& Jeuniaux, 2010). In line with the previous findings, the iconic configuration and reverse-iconic configuration can be viewed in individual's perception of a linguistic stimulus above or under the other or in the speed of response to the iconic or non-iconic linguistic stimulus (Zwaan \& Yaxley, 2003).

\section{Bodily Interaction and Perception of External Reality}

One of the most important events in cognitive studies in recent years has been the emergence of and the increasing interest in the notion of Embodied Cognition. With regard to "embodied embedded cognition (Clark 1997; Haselager 2004; Lakoff \& Johnson, 1999, cited in Kerkhofs \& Haselager, 2006), the "organism's bodily interaction with the environment is of crucial importance to its cognitive processes, both in relation to the kind of processes it engages in, as well as the way these processes are performed (Chiel \& Beer 1997)", (Kerkhofs \& Haselager, 2006, p. 2). On the word of this view, body has a central role in shaping the possibilities of shaping the cognitive tasks and solving them. Attributable to this perspective, the sensorimotor activities make the necessary interactions with the environment and shape the capacities for perception and meaning making.

To date there has been little attention to the effect of bodily actions on the perception of the meaning. However, in recent years, development in cognitive science has paid due attention to the process of meaning making. Meaning making, in this regard, is an unalike process, as well as a product, for various people because of the fact that it greatly depends on an individual's history and experience of interaction with the external world and as a result people may have various perceptions of the external reality depending on personal moment to moment interactions with the environment. To shed light on this view, Glenberg and Robertson (2000) draw our attention to the fact that "the meaning of a particular situation for a specific organism can be related to the coordinated set of actions that are available to it" (cited in Kerkhofs \& Haselager, 2006, p. 2). Therefore, embodied cognition is believed to be largely dependent on the bodily actions and as a result, perception and meaning making, which is assumed to be a cognitive action, can be regarded as a cooperated action between cognition and body movements.

Empirical research which has been done on the effect of body movements and cognition puts emphasis on the idea that an individual might simulate experience an action by mere thinking about a particular situation (Barsalou, 2002; Matlock et al., 2003). According to Matlock et al. (2003), an individual might make models of the external world by simulating the objects and movement, and make pathways to enrichment of the perception of physical world. 
One important point that accords with the Embodiment debate is the fact that each individual perceives a variety of the outside reality. Each individual explores the world and internalizes the experiences through perception and formulates the cognition. This can be the fundamental basis for the variety of the perception of and external reality by different individuals. It is hard to escape the idea that, despite the individual differences, such various experiences and as a result, numerous perceptions of an external reality may vary by cultural differences as well.

\section{Embodiment and Language Comprehension}

By drawing on the concept of Embodiment, Clark (2006, p. 370) points out that the role of language in making meaning is of extraordinary prominence, and it is due to the fact that words of language contain not only perceptual meaning but also materiality. Clark argues for the question "what is the cognitive role of language? Are words and sentences merely vehicles for the communication of preformed ideas, or are they part of the process of thinking itself?" Later, Clark (2006) proposes that "words and sentences form part of the process of thinking, and that they do so not merely in virtue of their contents but also in virtue of their very materiality: their physical existence as encountered and perceptible items, as sounds in the air or as words on the printed page" (p. 370). Clark (2006) holds that as a prerequisite for the comprehension of meaning, individuals construct structural representations of the environment which he calls “"cognitive niches' that enhance and empower us in a variety of nonobvious ways" (p. 370).

Findings in the realm of embodied cognition have been generalized to the language comprehension through which research has shown that understanding and making meaning of the linguistic stimulus is greatly dependent on one's perception of the external world. The theory of Embodiment in language comprehension has been elaborated by many scholars, among all, Glenberg and Kaschak (2003) found that the participants of the study, who were supposed to respond to the sentences on the screen of a computer and make feasibility judgments, were faster to accept the feasibility of a sentence and respond "yes" when the motor response and the described action matched.

Recent work on language comprehension enhances our understanding by highlighting two signified claims, namely Immersed Experiencer Framework (IEF) and the Indexical Hypothesis (IH). According to IEF, language user is assumed an immersed experiencer of the external reality which receives cues, or the language, which are provided for the language comprehender as the simulation of the external situation (Zwaan, 2004). On the other hand, owing to IH, the first step in comprehension is assumed to be indexing the language and the second step is to derive performances of each indexed objects and the third stage is to make a unified action of all the performances of the indexed objects (Glenberg, 1997).

Glenberg and Kaschak (2004) aims to give a comprehensive account of understanding a sentence which encompasses creating an action-oriented motor representation. As the writers maintain, when this creation of the action representation accords with a pre-existing motor representation, the process of the bodily movement would be, to a great deal, smoothed. In contrast to the condition of the compatibility of actions, if it conflicts with a previous action, it will hinder the process of the execution of the action. Therefore, the significance of the language as having a prominent role in activating perceptual representations as well as motor representations is highlighted which leads to giving escalation of an embodied depiction of linguistic comprehension.

\section{Embodiment and Conceptual Processing}

Currently, surveys inspire not only to the idea that Embodiment has a central role in conceptual processing but also to a more radical point that not much more to conceptual processing than the activation of embodied representations. Recently, the issue has been raised that whether conceptual processing is dependent on perceptual simulations or it is mere linguistic. Many research in this regard supported the fact that conceptual processing tasks involve both perceptual simulations and linguistic structure (Barsalou, Santos, Simmons, \& Wilson, 2008; Louwerse \& Jeuniaux, 2008; Zwaan, 2008). On the other hand, as cited in Louwerse and Jeuniaux (2010, p. 97), "some have argued that conceptual processing is predominantly embodied (Barsalou et al., 2008), whereas others have argued that it is predominantly linguistic in nature (Louwerse \& Jeuniaux, 2008)". Nevertheless, the idea is still vague. To provide ample evidence for the support of the above mentioned issue, Louwerse and Jeuniaux (2010) conducted four experiments to investigate the conditions under which Embodiment and linguistic factors dominated conceptual processes through which individuals made speeded judgments about the semantic or iconic interrelatedness of the words and pictures. These findings supported the fact that the results were modified by task, highlighting the significance of the strength of Embodiment factor in iconicity judgments for pictures and the significance of the strength of linguistic factor in semantic judgments for words. Both linguistic and Embodiment factors predicted error rates. In addition, the response time for both semantic and iconicity judgments were precisely evaluated. The results supported the duality of conceptual processing, being both linguistic and embodied, with a bias for the Embodiment or the linguistic factor depending on the nature of the task and the stimuli.

\section{Evidence}

The literature on Embodiment abounds with examples of three major evidence of appearance, which is derived from sentence-picture mapping, judgment and action description, and dynamic stimulus studies (Kaschak, 2005; Stanfield \& Zwaan, 2001; Zwaan, Stanfield, and Yaxley, 2002; Zwaan et al., 2004), evidence of affordance, which is obtained from afforded action versus non-afforded action studies (Gibson, 1979; Glenberg \& Robertson, 2000; Kaschak \& Glenberg, 2000), and evidence of action, which are concluded of the studies investigating the simultaneous action of the individual (Glenberg \& Kaschak, 2002) compatibility provide evidence in support of embodied cognition. 
A further specification of the Embodiment has been put forward as metaphoric mappings from the spatial (physical) to nonphysical domains. However, Sinha and Lopez (2000) concluded that "neither linguistic nor psycholinguistic evidence provides unequivocal support for the Embodiment thesis as it is usually formulated. That is to say, although the human body is (probably universally) a salient potential source schema for the linguistic conceptualization of space (and other domains), it is by no means the case that "bodily experience" (if by that is meant experience of one's own body) provides the sole or most common schematic basis for construing space, or for acquiring the language of space. If Embodiment is to remain (as we believe it should) a central notion in cognitive semantics, it requires a reformulation in terms which are psychologically (developmentally) realistic, and which link it more explicitly to systems of cultural schematization and understanding" (p. 23).

As put by Sinha and Lopez (2000), Embodiment, alternatively, can be viewed as the mutual relationships between language, culture, and cognition which have recently been systematically treated together in the modern psychological, linguistic and anthropological literatures.

\section{Embodiment Claims}

On a profounder contemplation on the embodied cognition, Wilson (2002) holds that within the debate of Embodiment, the cornerstone of the embodied cognition, six diverse claims need to be taken into consideration: 1. cognition is situated, 2. cognition is time pressured, 3. we off-load cognitive work onto the environment, 4. the environment is part of the cognitive system, 5. cognition is for action, and 6. off-line cognition is body based.

To justify what has been put forward by the Embodiment debated, Anderson (2003) upholds that as a result of rethinking in the nature of cognitive activity, cognition has been revitalized into an embodied or situated activity which is assumed to be a dramatic shift "from Descartes' "thinking thing", and the picture of human being and subjectivity it suggests, to a more Heideggerian approach to being in the world, in which agency and interactive coping occupy center stage, is an extremely important development, the implications of which are only just beginning to be fathomed" (p. 91).

As quoted by Wilson (2002), the situatedness of the cognition in the foundation in Embodiment debate. Situated cognition refers to the cognition required for the accomplishment of any task in a situated context of the task and through the coupling the cognition and the environment. Regarding the time pressured-ness of the cognition, any real time task which is contextually situated is limited with time boundaries. Claim three puts forward the idea that we offload cognitive work onto the environment. It basically means that, irrespective of times that individuals run cognitive processes offline, much of it is done online which lays a great burden on the individual on account of its demand. According to Wilson (2002), in these cases there are two kinds of options available: to relay on the background knowledge and/or to reduce the cognitive load by off-loading it to the environment as a cognitive strategy, "leaving information out there in the world to be accessed as needed, rather than taking time to fully encode it; and using epistemic actions (Kirsh \& Maglio, 1994) to alter the environment in order to reduce the cognitive work remaining to be done" (cited in Wilson, 2002, p. 628). In the fourth claim, the environment is assumed to be a containing part of the cognitive action. It means the internal to the individual and the environment assist cognitive activity. This idea is in line with the claim that cognitive activity is not limited to the brain and is a part of the environment which is in sharp contrast with the innatists or bahaviorists inclinations about the internally pre-specified or externally conditioned cognition. As put by Wilson (2002), "the claim is this: The forces that drive cognitive activity do not reside solely inside the head of the individual, but instead are distributed across the individual and the situation as they interact. Therefore, to understand cognition, we must study the situation and the situated cognition, together as a single, unified system" (p. 630). The fifth claim elaborates on the action relatedness of the cognition. That is according to Wilson (2002), "the function of the mind is to guide action, and cognitive mechanisms such as perception and memory must be understood in terms of their ultimate contribution to situation-appropriate behavior" (p. 626). And finally the sixth claim encompasses the idea that off-line cognition is body based, which means that it is greatly based on the external part of the coupled activity of the cognition and the environment which is greatly dependent on the moment to moment sensorimotor activities. Actually, according to Embodiment Theory, the cornerstone of the construction of the cognition is that "the activity of the mind is grounded in mechanisms that evolved for interaction with the environment—-that is, mechanisms of sensory processing and motor control" (Wilson, 2002, p. 626).

In summary, Wilson (2002) believes that it is better to treat the comprising claims of the Embodiment rather than treating the Embodiment Theory as a unified whole. This approach leads to achieving the advances and merits of each claim contributing to the Embodiment as a whole.

\section{Inheritable or Adventitious: The Role of the Environment}

Neisser's (1976, as quoted by Sinha and Lopez, 2000) establishes a relationship with Embodiment thesis with Gestalt psychology. It has also been demonstrated that there is a close association between Embodiment and Piaget's (1953) notions of sensorimotor stage as having a central and fundamental role in construction of cognitive structure of the child. As stated by Piaget, the child, from the early infancy, through experiencing the external reality internalizes the perceptual capability. However, Piaget was later criticized for his negligence in considering the prominent role of the cognitive-domain specificity of language, and of its possible formative role in cognitive development.

An alternative perspective has been adopted by and more utterly stated by Vygotsky $(1978,1986)$ from a socio-cultural approach. Vygotsky emphasized the noteworthy role of the environment and specificity of the interaction between the infant and the significant other in formulation of the cognitive structure of the human child. However, Vygotsky's 
perspective, "despite its many virtues, and its superiority to its formalist rival, it has failed to pay sufficient attention to the importance of culture and society in human cognition, in the motivation of linguistic structure, and in the acquisition of language" (Sinha \& Lopez, 2000, p. 19).

Closely related to the development of intelligence and deeply rooted in Vygostky's socio-cultural perspective toward the development of cognition, Embodiment is also in contrast with traditional views of intelligence as a fixed inherited underlying trait in each individual, irrespective of the environmental world. Smith and Gasser (2005, p. 13) maintain that "The central idea behind the Embodiment hypothesis is that intelligence emerges in the interaction of an agent with an environment and as a result of sensorimotor activity. This view stands in opposition to more traditional notions of internal representation and computation and in general has had little to say about symbols, symbolic reasoning, and language". Smith and Gasser (2005) offer six lessons for developing embodied intelligent agents suggested by research in developmental psychology. Accordingly, they hold the idea that "starting as a baby grounded in a physical, social, and linguistic world is crucial to the development of the flexible and inventive intelligence that characterizes humankind" (p. 13). According to Smith and Gasser (2005), there are six basic principles underlying the evidence which is prerequisite to support the Embodiment Theory. These principles include: a) the multimodality of the world which the baby is exposed to and experience from early infancy, b) the baby's non-premature initial state and their incremental maturation, c) the baby's exposure to the world endowed with regularities and their capability to construct perception, action, and ultimately thought through intelligence and the worlds' bootstrapping, d) the baby's inclination toward exploration, e) the baby's actions and learning through the mediation of the significant other, and finally f) the baby's symbolic use of language and communication which ultimately leads to higher level abstraction.

\section{Conclusion}

Early representations of an internal derive (Chomsky, 1959) or external derive (Skinner, 1957) seems to appear unsatisfactory to provide the whole sketch of the cognition and, as a result, the cognition seems to be locked internally away or reduced to external drive. The previous perspectives did not provide how the brain can be related to the external world and perception was assumed to be radically internal or basically external with a minor internally driven trigger. Due to this flaw in representation of the perception, cognitive scientists became more prone toward a way to represent the perception which is the mere relation of the mind with the external world as central and functional in a model of cognition in solving tasks which led to the progresses in perception-action research (Gibson, 1966, 1979). Shapiro (2011) calls this replacement hypothesis which is proposed when the previous hypothesis cannot meet the total requirements to represent the whole picture. "This hypothesis is inevitable once you allow the body and environment into the cognitive mix. If such replacement is viable, then, any research that keeps the standard assumptions of cognitive psychology and simply allows a state of the body to tweak cognition, misses the point" (Wilson \& Golonka, 2013, p. 2).

However, as put by Sinha and Lopez (2000), "the Embodiment thesis pays a kind of lip-service to the social surround, but does not explore its specific role in cognition and language, and tends to see cognitive mappings in terms of a oneway street from individual (embodied domains) to society (abstract and social domains)" (p. 19). Embodiment, basically, is dependent on the interactive nature of the organism with the environment as a social and physical one. "It does not, however, specify in which ways these two aspects of the organism's environment relate to each other; nor in what respects varying social environments may give rise to varying experiences; nor the extent to which such varying experiences may be relevant to the categories which are formed as a (partial) consequence of such experience" (Sinha \& Lopez, 2000, p. 19).

Driven by the inadequacies of the preceding hypothesis to account for the whole image of cognition, the emergence of a replacing hypothesis, as it was mentioned before, is inevitable. Within the realm of Embodiment there has been thrown up many investigation; nevertheless, despite the fact that the innovative hypothesis seems compensate the vacuity of the prior hypothesis; nonetheless, it lives at its early infancy and more information is required to establish a greater degree of accuracy on this matter.

\section{References}

Anderson, M. L. (2003). Embodied cognition: A field guide. Artificial Intelligence, 149, 91-130.

Barsalou, L. W. (1999). Perceptual symbol systems. Behavior and Brain Sciences, 22, 577-660.

Chomsky, N. (1959). Verbal behavior. Language, 35, 26-58.

Clark, A. (2006). Language, embodiment, and the cognitive niche. Trends in Cognitive Sciences, 10(8), $370-374$.

Damasio, A. (2000). The feeling of what happens: body and emotion in the making of consciousness. London: William Heinemann.

Elman, J., Bates, E., Johnson, M., Karmiloff-Smith, A., Parisi, D., \& Plunkett, K. (1996). Rethinking Innateness: A connectionist perspective on development. Cambridge: MIT Press/Bradford Books.

Gibbs, R. W., Jr. (2003). Embodied experience and linguistic meaning. Brain and Language, 84, 1-15.

Gibson, J.J. (1966). The senses considered as perceptual systems. Boston: Houghton Mifflin.

Gibson, J. J. (1979). The ecological approach to visual perception. Boston: Houghton-Mifflin.

Glenberg, A. M. (1997). What memory is for. Behavioral and Brain Sciences, 20, 1-55. 
Glenberg, A. M., \& Kaschak, M. P. (2002). Grounding language in action. Psychonomic Bulletin \& Review, 9, 558-565.

Glenberg, A.M., \& Kaschak, M.P. (2003). The body's contribution to language. The Psychology of Learning and Motivation, 43, 93-126.

Glenberg, A. M., \& Kaschak, M. P. (2004). Language is grounded in action. In L. Carlson \& E. van der Zee (Eds.), Functional features in language and space: Insights from perception, categorization, and development (pp. 11-24). Oxford: Oxford University Press.

Glenberg, A. M., \& Robertson, D. A. (2000). Symbol grounding and meaning: A comparison of high-dimensional and embodied theories of meaning. Journal of Memory and Language, 43, 379-401.

Harnad, S. (1990). The symbol grounding problem. Physica, D, 42: 335-346.

Kaschak, M. P., \& Glenberg, A. M. (2000). Constructing meaning: The role of affordances and grammatical constructions in sentence comprehension. Journal of Memory and Language, 43, 508-529.

Kerkhofs, R., \& Haselager, W.F.G. (2006). The embodiment of meaning. Manuscrito, 29(2), 753-764.

Louwerse, M. M., \& Jeuniaux, P. (2008). Language comprehension is both embodied and symbolic. In M. de Vega, A. Glenberg, \& A. C. Graesser (Eds.), Symbols, embodiment, and meaning (pp. 309-326). Oxford: Oxford University Press.

Louwerse, M. M. \& Jeuniaux, P. (2010). The linguistic and embodied nature of conceptual processing. Cognition, 114, 96-104.

Pecher, D., \& Zwaan, R. A. (Eds.). (2005). Grounding cognition: The role of perception and action in memory, language, and thinking. New York: Cambridge University Press.

Plunkett, K., \& Sinha, C. (1992). Connectionism and developmental theory. British Journal of Developmental Psychology, 10, 209-254.

Semin, G. R., \& Smith, E. R. (Eds.). (2008). Embodied grounding: Social, cognitive, affective, and neuroscientific approaches. New York: Cambridge University Press.

Shapiro, L. (2011). Embodied cognition. NewYork: Routledge Press.

Sinha, C., \& López, K.J. (2000). Language, culture and the embodiment of spatial Cognition. Cognitive Linguistics, 11, $17-41$.

Smith, L., \& Gasser, M. (2005). The development of embodied cognition: Six lessons from babies. Artificial Life, 11, $13-29$.

Skinner, B.F. (1957). Verbal Behavior. NewYork: Appleton-Century- Crofts.

Stanfield, R. A., \& Zwaan, R. A. (2001). The effect of implied orientation derived from verbal context on picture recognition. Psychological Science, 13, 153-156.

Vygotsky, L. (1978). Mind in Society: the development of higher psychological processes. In C. Michael, V. JohnSteiner, S. Scribner \& E. Souberman (Eds.). Cambridge: Harvard University Press.

Vygotsky, L. (1986). Thought and language. In A. Kozulin (Ed.). Cambridge: Harvard University Press.

Wilson, M. (2002). Six views of embodied cognition. Psychonomic Bulletin \& Review, 9 (4), 625-636.

Wilson, A. D., \& Golonka, S. (2013). Embodied cognition is not what you think it is. Frontiers in Psychology, 4(58), 113.

Zwaan, R. A. (2004). The immersed experience: Toward an embodied theory of language comprehension. In B. H. Ross (Ed.), The psychology of language and motivation (Vol. 44). New York: Academic Press.

Zwaan, R. A., Madden, C. J., Yaxley, R. H., \& Aveyard, M. E. (2004). Moving words: Dynamic representations in language comprehension. Cognitive Science, 28, 611-619.

Zwaan, R. A., Stanfield, R. A., \& Yaxley, R. H. (2002). Language comprehenders mentally represent the shapes of objects. Psychological Science, 13, 168-171.

Zwaan, R. A., \& Yaxley, R. H. (2003). Spatial iconicity affects semantic relatedness judgments. Psychonomic Bulletin and Review, 10, 954-958. 